\title{
Preliminary Study of Launch and Orbit of a CubeSat Using a Modified VSB-30 Launcher Vehicle
}

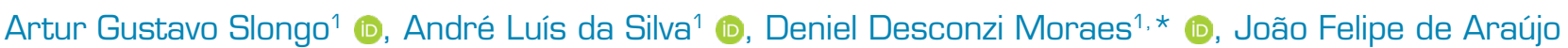 \\ Martos $^{1}$ (D), Lorenzzo Quevedo Mantovani ${ }^{1}$ (D), Mateus Schmitz Venturini (D), Róger Mateus Sehnem ${ }^{1}$ (D)
}

1. Universidade Federal de Santa Maria - Centro de Tecnologia - Departamento de Engenharia Mecânica - Santa Maria/RS - Brazil.

*Correspondence author: d.desconzi@gmail.com

\section{ABSTRACT}

During the last decade, the world faced the mass insertion of small satellites in the space technology scenario. Every year, the number of micro and nanosatellites launched increases and gets more attention from players in the space market. Despite the lack of a national launcher, the Brazilian Space Program is known for some successful development in the last century, including its space assets, such as a privileged launch site near the equator, a family of flight proven and reliable sounding rockets for suborbital flights and microgravity experiments and universities with established small satellites programs. Thereby, the present work proposes a modification of the Brazilian VSB-30 sounding rocket in order to allow the launch and insertion in low Earth orbit (LEO) of small satellites fulfilling the gap of a national launcher. It also presents a CubeSat orbital decay simulation and orbital insertion simulation with the modified rocket launched from the Alcântara Launch Center as a matter of verifying the potential of national missions using this modified launcher.

Keywords: Flight simulation; Orbit calculation; Orbit decay; Small scientific satellites; Sounding rockets.

\section{INTRODUCTION}

During the last decade, the world faced the mass insertion of small satellites in the space technology scenario. The dynamism of these projects, its low relative cost for manufacturing, testing and launching, as well as its integration building potential and support for scientific research and technological development brought CubeSats and other small satellites into evidence (Wekerle et al. 2017). The presence of small satellites in the space industry has increased to such a degree that, in 2017, the number of these satellites launched was greater than the conventional satellites, in accordance with the Brazilian Center of Management and Strategic Studies (CGEE 2018). Furthermore, according to O'Donnell and Richardson (2020), 1364 small satellites $(<500 \mathrm{~kg}$ ) were successfully launched between the years of 2009 and 2018 . From those satellites, 968 (71\%) were either PicoSats $(<1 \mathrm{~kg})$ or NanoSats $(1-10 \mathrm{~kg})$, the latest being responsible for 804 of the successful launches.

In Brazil, the initiative for launching CubeSats started at the National Institute for Space Research (INPE) in partnership with the Federal University of Santa Maria (UFSM). The Brazilian INPE-UFSM NANOSATC-BR, CubeSats Development Program launched the first Brazilian CubeSat, NANOSATC-BR1 (1U) in 2014. Additionally, the program has been developing their second

Received: Feb. 10, 2020 | Accepted: Oct. 10, 2020

Peer Review History: This is a peer reviewed version of selected paper presented at the $2^{\circ}$ Congresso Aeroespacial Brasileiro occurred in 16 -19 September of 2019 at Universidade Federal de Santa Maria, Santa Maria/RS, Brazil.

Section Editor: Marcelo Serrano Zanetti

This is an open access article distributed under the terms of the Creative Commons license. 
and third CubeSats, NANOSATC-BR2 (2U) and NANOSATC-BR3. According to the Brazilian Space Agency (AEB 2020), the NANOSATC-BR2 is expected to be launched in November 2020. Other initiatives include the Aeronautics Institute of Technology (ITA) first CubeSat, ITASAT-1 (6U), launched in 2018 (Sato et al. 2019). Moreover, their ongoing ITASAT-2 project, kicked off in July 2020 (ITA 2020).

Regarding the launch of these vehicles, usually they are secondary payloads on heavy launch systems, but there is a new market for dedicated small satellites launcher. As an example, the United States Department of Defense and NASA funding programs follow the idea of funding not just traditional launch services but also "launch-on-demand" systems, in view of providing both incentive and guidance for new fledgling companies. Additionally, the United Kingdom and China are also focusing their research on the development of small satellite space projects (Niederstrasser and Madry 2020), which contribute to the research and development of dedicated launchers for these vehicles.

In addition, Niederstrasser and Madry (2020) mention that, since the launch of the Jielong 1 launcher in April of 2019, there are currently eight operational systems capable of carrying payloads of less than $1000 \mathrm{~kg}$ to low Earth orbit (LEO). Five of those eight are Chinese vehicles, being the most recent ones Hyperbola-1 and the already mentioned Jielong 1, from iSpace and China Rocket Co. Ltd, respectively. The Chinese operational systems are expected to be commercially offered outside of China eventually, even though it has not yet happened. In the non-Chinese vehicles category, there are the older ones in activity, Pegasus XL and Minotaur I from Northrop Grumman, and the newest western launcher, the Rocket Lab Electron, which has had a $100 \%$ success rate since its maiden flight failure in 2017 (Niederstrasser and Madry 2020). Furthermore, a myriad of other small satellites launchers is in development or funding phases (Niederstrasser 2018).

In accordance with the presented trend, this work proposes the feasibility analysis of launching a CubeSat from a modification of the Brazilian sounding rocket VSB-30. It also presents a CubeSat orbital decay simulation, after VSB-30 modification orbital insertion, as a matter of verifying the potential of missions using the proposed launcher.

\section{Brazilian Space Assets}

The development of space projects in Brazil started with the Sonda rocket family with SONDA I, launched in 1965, followed by SONDA II, launched in 1970, SONDA III, launched in 1976, and SONDA IV, launched in 1984. The Sonda rocket family was launched at the Barreira do Inferno Launch Center (CLBI), located near Natal, capital of Rio Grande do Norte (Palmerio 2017). The main objective of the Sonda family was to be the base of a Brazilian research and development space project. Additionally, the lessons learned, some of the developed technologies and even parts of the previous rockets are used with each new rocket. For example, S20, motor from SONDA II, is the second stage of SONDA III; S30, motor of the first stage of SONDA III, is the second stage of SONDA IV. The research and development acquired from the SONDA rockets led to the projects of VLS and eventually VSB-30, both followed the idea of using former developed technologies, for instance, the motors S20 in the first two versions of VLS and S30 used on the second stage of VSB-30 (Palmerio 2017). Unfortunately, since the VLS accident in 2003, the Brazilian space project did not continue with the same grit as before.

Adding to the active Brazilian space assets, there are the projects of CubeSats by INPE and ITA, as previously mentioned. Furthermore, universities all over Brazil have been working towards student programs for space research, either for competitions or for academic purposes, as this is a low-cost technology to be implanted in universities. Research in the aerospace field has also been encouraged recently by the creation of the Brazilian Aerospace Congress (CAB) in 2018.

Another driver for a space mission proposal to take place in Brazil is the Alcântara Launch Center (CLA). Located near the city of Alcântara, in the state of Maranhão, in a privileged location, $2^{\circ} 18^{\prime}$ south of the Equator, the CLA is a known Brazilian asset in the space industry. As stated by the Department of Science and Aerospace Technology (DCTA 2019), the region has positive characteristics for launchings.

The advantages that justify the use of this launch center in the defined national mission proposal are, among others:

- Fuel saving for low inclination orbits when compared to centers located in high latitudes; 
- Favorable safety conditions for launches on trajectories towards polar, inclined and equatorial orbits since the launch vehicle can avoid inhabited areas with small need of correction maneuvers;

- Favorable climatic conditions, with a well-defined rainfall regime and tolerable winds, in addition to small temperature variations throughout the year;

- Geological stability;

- Good logistic support due to its proximity with São Luís, Maranhão’s capital city;

- Use and appreciation of installed national facilities;

- Support from trained and national technical staff.

\section{Modified VSB-30 Launch vehicle}

The VSB-30 is a two-stage, unguided and rail launched sounding vehicle. The vehicle consists of a solid propellant S31 rocket booster, a boost adapter, the second stage S30, payload, a recovery and a service system (Garcia et al. 2011). The vehicle is designed to fly in a spin stabilized unguided mode. The VSB-30 is an adaptation proposed by the German Aerospace Center to substitute the British vehicle, Skylark 7, on the European microgravity program (Palmerio 2017). The VSB-30 is currently used mainly as a sounding vehicle in order to perform experiments in microgravity through suborbital flights. It can deliver up to $400 \mathrm{~kg}$ of payload at $250 \mathrm{~km}$ of altitude, allowing $350 \mathrm{~s}$ of microgravity environment. It has an apogee of $250 \mathrm{~km}$ and its dispersion point has a radius of $50 \mathrm{~km}$ from its launch center (Garcia et al. 2011; Palmerio 2017).

Within the scope of this work is the suggestion of modifications to this vehicle turning its service module in a third stage, in order to allow the launch and insertion in LEO of small satellites. Hence, the modified VSB-30 (VSB-30M) can be defined as a three-stage vehicle, with two solid-propellant engines and a third engine that should deliver the necessary amount of extra thrust necessary to achieve a stable orbit. The development of the third stage was done according to a number of requisites and references described throughout this work. The proposed modification of the VSB-30 is based on an analysis of small satellite launch vehicles currently in operation or development. Moreover, the number of stages to be added shall follow a trade-off between increment of performance and complexity, so the minimum number of stages that accomplish mission requirements shall be selected (Sutton and Biblarz 2001).

Figure 1 shows the current configuration of the launcher, and Table 1 presents the characteristics of each stage.

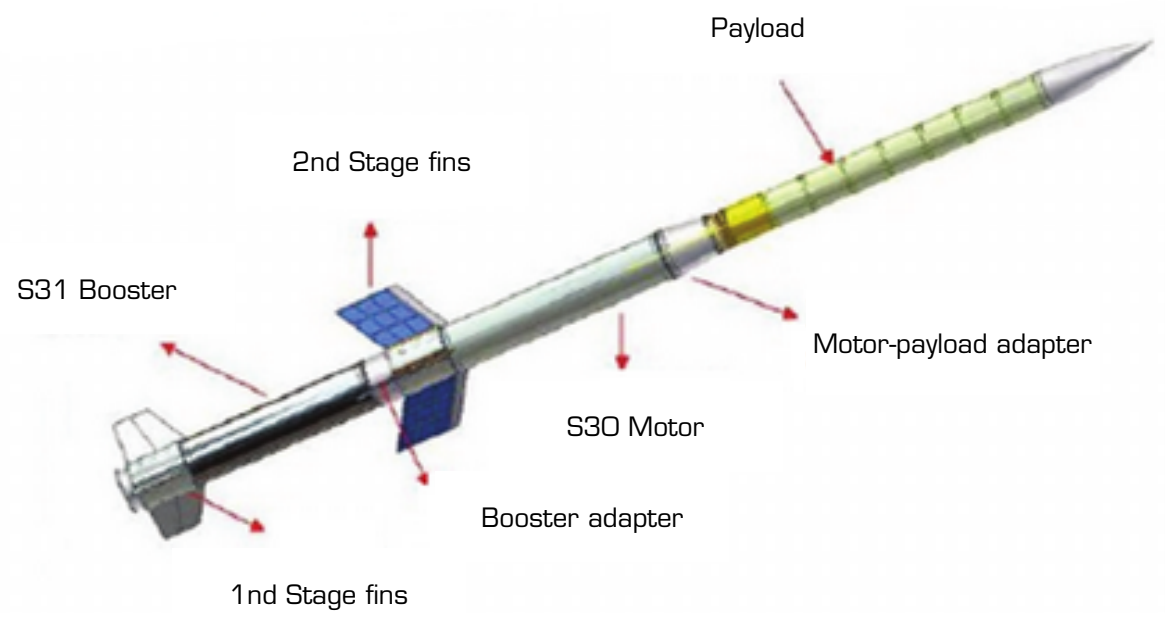

Figure 1. VSB-30 launch vehicle. Adapted from Silva et al. (2013). 
Table 1. VSB-30 original parameters.

\begin{tabular}{cccc}
\hline Parameters & First stage (S31) & Second stage (S30) & Service module \\
\hline Specific impulse (in vacuum) & $260 \mathrm{~s}$ & $260 \mathrm{~s}$ & \\
Structural mass & $240 \mathrm{~kg}$ & $250 \mathrm{~kg}$ & $400 \mathrm{~kg}$ \\
Propellant ratio & 0.7363 & 0.7778 & \\
Propellant mass & $670 \mathrm{~kg}$ & $875 \mathrm{~kg}$ & \\
\hline
\end{tabular}

Adapted from Palmerio [2017).

\section{METHODOLOGY}

\section{Launch Vehicle Parameters}

The propulsive model used for the first two stages is obtained from Palmerio (2017), while the parameters of the third stage are estimated from other vehicles that fulfill the envisaged mission for the VSB-30M. An exception is made in determining the structural ratio (SR) of the third stage of the VSB-30M, which was defined after the analysis of the Tsiolkovsky equation results and feasible values given in the literature.

Other features of the third stage are the result of a simplified review of commercial launchers (2019 Review of Commercial Launchers of Small Satellites by Moraes D. D.; unreferenced, see "Acknowledgments" section) and the data for the ISAS/JAXA modified SS-520 vehicle, or SS-520-5 (Inatani and Ohtsuka 2018). The data acquired from the commercial launchers review is compared with the values that solve the Tsiolkovsky equation for the $\Delta V$ presented in the next section. For the commercial review, 49 small satellite launch vehicles were evaluated looking for the following characteristics:

- That the vehicle could reach the 3-stage configuration;

- That the vehicle had a third liquid stage.

Focus was given to determining the following characteristics of the third stages:

- Propellants used and their characteristics;

- Specific impulse;

- Ratio of inert mass to mass of propellant;

- Thrust;

- General physical dimensions and diameter of the service modules;

- General engine information.

- Reliable and recent data from each project.

From 49 launchers only launchers that fulfill the previous requisites are presented in Table 2, which summarizes some data of interest about the launchers that fit the requirements given above, in the case of rocket families, a model was chosen for representation.

The main source of data for determining characteristics of the third stage was the JAXA SS-520-5 launcher. As described by Inatani and Ohtsuka (2018), this small satellite launcher is a three-stage rocket with "SS-520s two stage rocket" and a "small third solid rocket motor housed in a fairing". Its configuration and development history are in accordance with the proposal of this work, as a result of the modification of a two-stage, flight proven, sounding rocket by adding a third stage in view of inserting small satellites into Earth's orbit. Furthermore, the SS-520-5 successfully launched and delivered a 3U CubeSat into orbit in January 2018. This achievement reached by ISAS/JAXA, makes the SS-520-5 the likely best benchmark for improvements of sounding rockets to becoming small satellite launchers. Thus, it is used as the reference for data on solid third stage rockets and the characteristics of interest are shown in Table 2.

The term diameter of interest (DI) represents the diameter of the third stage structure, while $I_{s p}$ indicates the specific impulse; $T$, in turn, refers to the thrust generated by the engine. While total mass (TM) is linked with the complete launch vehicle. 
Table 2. General information about small satellite launch vehicles for decision making about the VSB-30M.

\begin{tabular}{|c|c|c|c|c|c|c|c|c|}
\hline Launcher & $\begin{array}{l}\text { Payload } \\
\text { [kg] }\end{array}$ & $\begin{array}{l}\text { Orbit } \\
{[\mathrm{km}]}\end{array}$ & $\begin{array}{c}\text { DI } \\
\text { [m] }\end{array}$ & $\begin{array}{l}\text { TM } \\
{[\mathrm{kg}]}\end{array}$ & $\begin{array}{c}\text { Vacuum } I_{s p}[\mathbf{s}] \\
\text { [3 }{ }^{\text {rdd }} \text { Stage] }\end{array}$ & $\begin{array}{c}T[\mathrm{kN}] \\
\text { [3 }^{\text {rd }} \text { Stage] }\end{array}$ & Propellant & $\begin{array}{c}\text { Engine } \\
\text { [ } 3^{\text {rd }} \text { Stage] }\end{array}$ \\
\hline Taymyr-1A & 12 & - & $\sim 0.45$ & 2600 & 187-224 & 3.92 & $\begin{array}{l}\text { Hydrogen peroxide (HTP) } \\
\text { (PV-85) and aviation } \\
\text { kerosene (TS-1) }\end{array}$ & RLD-100s \\
\hline Electron & 150 & 500 & 1.2 & 12500 & N/D & 0.12 & - & Currie \\
\hline Skyora XL & 320 & 600 & - & - & 290-306 & 7 & $\begin{array}{l}\text { Hydrogen peroxide (HTP) } \\
\text { and kerosene (RP-1) }\end{array}$ & - \\
\hline Bloostar & $75-140$ & $\begin{array}{r}200 \\
-600\end{array}$ & - & 5818 & 355 & - & $\begin{array}{l}\text { Liquid methane and } \\
\text { oxygen }\end{array}$ & Teide 1 \\
\hline $\begin{array}{l}\text { Neptune } \\
\text { N1 }\end{array}$ & 20 & 500 & $\sim 0.64$ & 2449 & $245-300$ & 3.3-4.4 & $\begin{array}{l}\text { Steaming nitric acid } \\
\text { (WFNA) and turpentine }\end{array}$ & $\begin{array}{c}\text { GPRE } \\
0.75 K N T A\end{array}$ \\
\hline SS-520-5 & 4 & - & 0.52 & 2579 & 282.6 & 12.76 & BP-211J & - \\
\hline
\end{tabular}

Based on this analysis, two modifications were proposed. One considering a liquid third stage and another considering a solid third stage. It is worth mentioning that the solid motors technology is well known in Brazil, which may make it the best alternative for this modification, since it would be possible to develop the third stage solid rocket with national technology. While, the commercial development of liquid rocket engines is not fully dominated in Brazil, developing the third stage as a liquid rocket engine could be considered as an effort to the development of Brazilian technology.

The liquid third stage should be composed of, at least, a nose cone, Pico Orbital Deployer (POD), fuel tanks, injector, pumps, feed system, valves, combustion chamber and nozzle. Similarly, the solid third stage should be composed of, at least, a nose cone, POD, case, grain and nozzle. Their total mass is defined as the same as the original service module for calculation purpose. The main characteristics for each of them are summarized in Table 3.

\section{Tsiolkovsky Equation}

The Tsiolkovsky equation establishes the speed gain of rockets, the equation relates the maximum change of velocity of the rocket, $\Delta V$, to the effective exhaust velocity, which is a product of the specific impulse and Earth's gravitational acceleration at sea level, $I_{s p} g_{0}$, and the initial and final mass of the rocket, $M_{0}$ and $M_{b}$, respectively. Since there are three stages on the VSB-30M, the equation is developed for multiple stages, that way, as each stage burns, they are discarded. Thus, the equation is written as a sum of $\Delta V$ achieved by each stage. The Tsiolkovsky equation for three stages is shown in Eq. 1 :

$$
\Delta V_{\text {total }}=\sum_{i=1}^{3} I_{s p}(i) g_{0} \ln \left(\frac{M_{0}(i)}{M_{b}(i)}\right) .
$$

Both initial and final mass for each of the stages are calculated as a function of the propellant mass $\left(M_{p}\right)$, consumed during the flight, structural mass $\left(M_{s}\right)$ of each stage, discarded after the burnouts and payload mass $\left(M_{l}\right)$ for each stage, which considers nonburned stages as payloads for the first ones. Thus, the interest mass for a stage after and before burnout is defined in Eqs. 2-4, as follows:

$$
\begin{gathered}
M_{0}(i)=M_{s}(i)+M_{p}(i)+M_{l}(i) ; \\
M_{b}(i)=M_{s}(i)+M_{l}(i) ; \\
M_{l}(i)=M_{s}(i+1)+M_{p}(i+1)+M_{s}(i+2)+M_{p}(i+2)+\cdots+M_{s}(i+k)+M_{p}(i+k)+M_{l} .
\end{gathered}
$$

where $i$ is the stage number and $k$ is the last stage number. 
To guarantee a LEO orbit delivering, the first necessary step is to compare the needed $\Delta V$ for achieving these orbits and the $\Delta V$ generated by the rocket (delta- $\mathrm{V}$ budget).

Starting from the mass characteristics of the original VSB-30 and the minimum value of $\Delta V$ to reach LEO accounting for disturbance losses, defined by Ley et al. (2009) as $9300 \mathrm{~m} / \mathrm{s}$, it is necessary to evaluate a feasible $I_{s p}$ of the third stage and the fractions of the service's module $400 \mathrm{~kg}$ that are dedicated to propellant, payload and structure. The payload mass was chosen as the upper mass limit for defining a satellite as a CubeSat, $10 \mathrm{~kg}$. The $I_{s p}$ was chosen regarding the data presented in Table 2. In addition, the $9300 \mathrm{~m} / \mathrm{s} \Delta \mathrm{V}$ value was corrected to $9500 \mathrm{~m} / \mathrm{s}$ to ensure higher LEO orbit insertion. Finally, with the inputs the propellant and structure mass were calculated with the aid of the Tsiolkovsky equation. The resultant third stage mass characteristics were then transformed in dimensionless coefficients, in order to validate the gathered results by comparison with the values in literature. Equations $5-8$ show the calculation of the payload ratio $(\lambda)$, structural coefficient $(\epsilon)$, mass ratio $(\mu)$ and propellant ratio $(\zeta)$.

$$
\begin{gathered}
\lambda(i)=\frac{M_{0}(i+1)}{M_{0}(i)-M_{0}(i+1)} \\
\epsilon(i)=\frac{M_{S}(i)}{M_{0}(i)-M_{0}(i+1)} \\
\mu(i)=\frac{\epsilon+\lambda}{1+\lambda} \\
\zeta=1-\epsilon
\end{gathered}
$$

At last, Table 3 presents the discussed data for each of the proposed modifications.

Table 3. VSB-30M third stage.

\begin{tabular}{ccc}
\hline VSB-30M parameters & Solid rocket third stage & Liquid rocket third stage \\
\hline Specific Impulse (in vacuum) & $282.6 \mathrm{~s}$ & $305 \mathrm{~s}$ \\
Total mass & $400 \mathrm{~kg}$ & $400 \mathrm{~kg}$ \\
Structural mass & $29.29 \mathrm{~kg}$ & $35.4976 \mathrm{~kg}$ \\
Propellant ratio & 0.9249 & 0.9090 \\
Propellant mass & $360.71 \mathrm{~kg}$ & $354.5024 \mathrm{~kg}$ \\
\hline
\end{tabular}

For medium-sized solid motors (45-450 kg), the maximum propellant ratio according to Sutton and Biblarz (2001) is around 0.91 . As seen in Table 3 , for a third solid stage, the propellant ratio is a bit over the maximum value in the literature. However, as this parameter is used to make approximate and preliminary design estimates, it was decided to not discard this modification already. According to Sutton and Biblarz (2001), a high value of $\zeta$ indicates a low inert motor mass, an efficient design of the hardware, and a high stress. Values over the maximum can be reached using top-level materials and technologies during the development of the motors. In addition, achieving the third liquid stage propellant ratio is feasible in accordance with the data presented in Costa and Vieira (2010), retrieved from Isakowitz et al. (1999), which presents motors such as the Chinese YF-40 $(\zeta=0.93)$, the Russian 11D49 $(\zeta=0.93)$, the American RS27A $(\zeta=0.93)$ and the Soviet $\mathrm{RD}-171(\zeta=0.92)$. 


\section{Gravity Turn Maneuver}

The launcher simulation considers a gravity turn maneuver to insert the satellite in Earth's orbit. In this kind of orbit insertion, the orbit is achieved solely by carefully choosing both the launch direction and the waiting times direction and the waiting times before igniting each of the rockets engines after its preceding engine burnout. In this way, for a three-stage rocket, the orbit is achieved by using the first two stages subsequently (i.e., with the burnout of the first stage close to the ignition time of the second stage), and then, after the second burnout, waiting for the gravity turn effect to bring the flight path angle $\phi$ to zero. When this is achieved, the third stage is ignited and then, the gross part of the third stage energy is used to gain the necessary orbital velocity, while the first two are mainly used for gaining altitude and exiting the denser initial atmosphere.

\section{MATHEMATICAL MODELLING}

\section{Reference Systems}

The modelling of any dynamic behavior depends on the reference system used. In this work, four different coordinate systems compose the complete reference system utilized for modelling of the transatmospheric and space flights of the launch vehicle and orbit analysis of the satellite.

The first essential reference frame is called the inertial reference frame and is defined as the frame where the absolute acceleration is equal to zero. Even though such a frame does not exist in reality, a fixed coordinate system with negligible acceleration can be defined as an inertial frame of reference.

For Earth orbits, the inertial frame is traditionally defined as a coordinate system fixed with respect to distant stars. This reference frame is known as celestial reference system $\left(S X_{i} Y_{i} Z_{i}\right)$ or Earth centered inertial (ECI). The ECI is fixed at the Earth's center of mass, with the $S X_{i}$ axis pointing towards the vernal equinox, and the $S Z_{i}$ axis normal to the Earth's ecliptic plane. The $S Y_{i}$ axis completes the orthogonal coordinate system. Another description of this frame of reference is by means of the unit vectors $I_{i}, J_{i}$, $K_{i}$, which are parallel to $S X_{i}, S Y_{i}$ and $S Z_{i}$ axis, respectively.

During launch maneuvers and in low earth orbits, the atmospheric influences are not negligible. Since the atmosphere moves with Earth's surface, it is convenient to define a frame of reference with the same angular velocity as the Earth. This frame of reference is called Earth-centered, Earth-fixed (ECEF). Their axes are SX, SY and $S Z$ pointing towards the $I$, $J$ and $K$ unity vectors, respectively. The origin of the ECEF coincides with the ECI's origin. The SZ axis points towards the true north and is coincident to the $S Z_{i}$ axis. The position of SX and SY axes is obtained through a rotation of $\gamma_{G}+\omega_{e} t$ radians around the $S Z_{i}$ axis, where $\gamma_{G}$ is the initial angular position, $\omega_{e}$ is Earth's angular velocity and $t$ is the transcribed time with respect to the reference time $t_{0}$.

The direction cosine matrix that relates the ECEF and the ECI frames is shown in Eq. 9.

$$
C_{E C E F}=C_{3}\left(\gamma G+\omega_{e} t\right)=\left(\begin{array}{ccc}
\cos \left(\gamma G+\omega_{e} t\right) & -\sin \left(\gamma G+\omega_{e} t\right) & 0 \\
\sin \left(\gamma G+\omega_{e} t\right) & \cos \left(\gamma G+\omega_{e} t\right) & 0 \\
0 & 0 & 1
\end{array}\right)
$$

In orbital dynamics, it is more convenient to express movement and position with spherical coordinates. A spherical coordinate system can be deduced from the ECEF reference frame using the radial distance $r$, and the $\delta$ and $\lambda$ angles, which represent, respectively, the orbit radius, celestial latitude and celestial longitude.

The next reference frame is defined in order to specify the velocity $v$. It is called the local horizontal vertical (LHV), denoted by oxxyz. The $o x$ axis points towards the radial distance vector, the $o z$ axis points towards the true north, and the $o y$ axis completes 
the orthogonal system pointing towards the east. The unity vectors which represent the LHV reference system are $i, j$ and $k$. The LHV system is related to the ECI system through the rotation matrix shown in Eq. 10.

$$
C_{L H}=C_{2}\left(\frac{\pi}{2}-\delta\right) C_{3}(\lambda)=\left(\begin{array}{ccc}
\cos \delta \cos \lambda & \cos \delta \sin \lambda & \sin \delta \\
\sin \lambda & \cos \lambda & 0 \\
-\sin \delta \cos \lambda & -\sin \delta \sin \lambda & \cos \delta
\end{array}\right) \text {. }
$$

Finally, a reference frame parallel to the velocity vector is adopted in order to handle the influence of aerodynamic variables in the atmospheric flight dynamics. This frame is called the Aerodynamic Reference Frame. No turbulent wind models or components are modelled. Thus, the direction of the wind velocity vector is idealized as parallel to the body's velocity relative to the ECEF frame, $v$. This frame has axes $S x_{v}, S y_{v}$ and $S z_{v}$ pointing towards the $i_{v}, j_{v}$ and $k_{v}$ unity vectors, respectively. The first presented axis is written along the instantaneous, relative velocity vector, $v$, defined previously. The remaining axes are normal to $v$ and mutually perpendicular. Those three axes are called the wind axes.

Figure 2 presents the relationships between the ECI, ECEF and LHV frames. Figure 3 shows the LHV and aerodynamic frames.

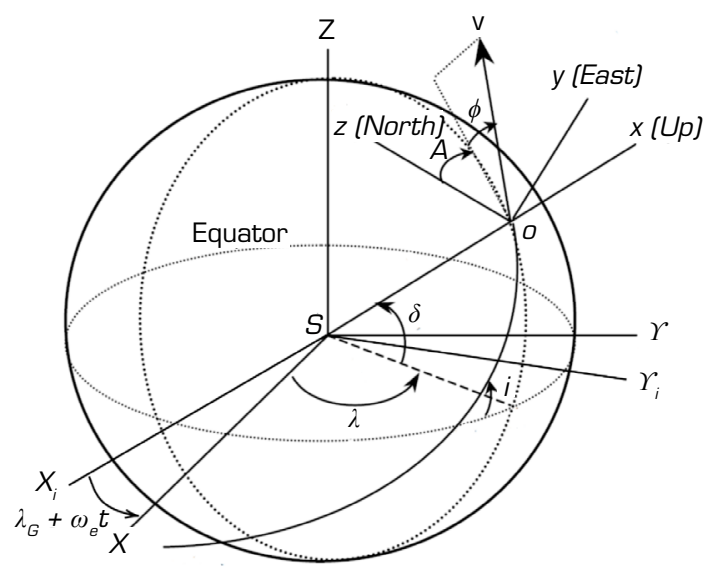

Figure 2. The ECI, ECEF and LHV systems. Adapted from Tewari (2007).

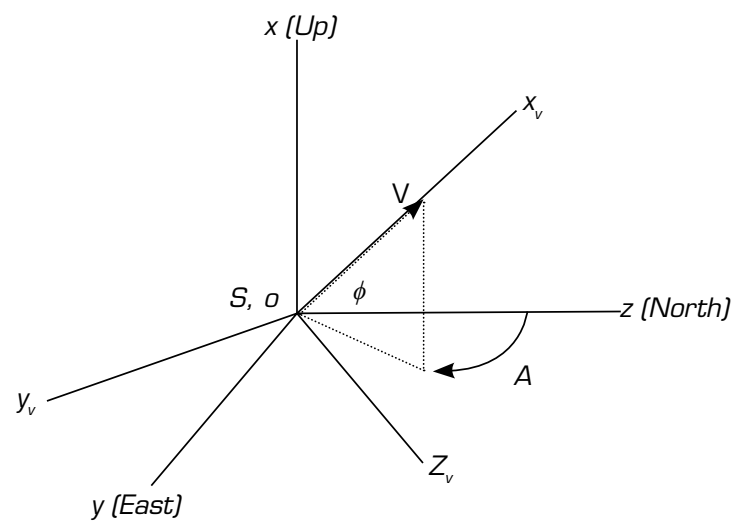

Figure 3. The LHV and aerodynamic coordinate system. Adapted from Tewari (2007). 
The last reference frame is useful to write the satellite equations. It is called the local vertical local horizontal (LVLH) reference frame in which $S Z_{L V L H}$ points towards the nadir, $S Y_{L V L H}$ axis is normal to the orbit plane and $S X_{L V L H}$ completes the orthogonal system (WIE 2008).

\section{Equations of Motion}

The translational motion of a body is completely described by three dynamic equations and three kinematic equations. The kinematic equations define the motion of the body without reference to the forces that cause the motion. The dynamic equations describe the relation between the forces applied at the system and its acceleration.

\section{Launch Vehicle}

The kinematic equations shown in Eq. 11 are deducted in Tewari (2007) for a rotating planet and constitute the first part of the set of six dynamic equations that describe the translational motion of the launch vehicle.

$$
\left\{\begin{array}{c}
\dot{r}=v \sin \phi \\
\dot{\delta}=\frac{v}{r} \cos \phi \cos A \\
\dot{\lambda}=\frac{v \cos \phi \sin A}{r \cos \delta}
\end{array}\right.
$$

Besides the previously defined variables, this set of equations also contains the variables flight path angle $(\phi)$ and velocity azimuth $(A)$. Opportunely, the up dot defines, as usual, a derivative.

For the launch vehicle, the acting forces have an aeropropulsive component that is written directly in the aerodynamic frame and a weight component due to gravity, given in the LHV frame and represented in the aerodynamic frame through a rotation matrix. In addition, the application of Newton's second law in a noninertial reference frame generates virtual forces due to the angular velocities between the frames of reference. The dynamic equations at the aerodynamic frame for the launching vehicle are shown in Eqs. 12-14 and are given by Tewari (2007). They define the dynamic motion of a body during transatmospheric flight such as reentry or launching.

$$
m \dot{v}=f_{T} \cos \epsilon \cos \mu-D-m g_{c} \sin \phi+m g_{\delta} \cos \phi \cos A-m \omega^{2} r \cos \delta(\cos \phi \cos A \sin \delta-\sin \phi \cos \delta),
$$

$$
\begin{aligned}
& m v \cos \phi \dot{A}=m \frac{v^{2}}{r} \cos ^{2} \phi \sin A \tan \delta+f_{T} \sin \mu+f_{Y}-m g_{\delta} \sin A+m \omega^{2} r \sin A \sin \delta \cos \delta- \\
& 2 m \omega v(\sin \phi \cos A \cos \delta-\cos \phi \sin \delta) \\
& m v \dot{\phi}=m \frac{v^{2}}{r} \cos \phi+f_{T} \sin \epsilon \cos \mu+L-m g_{c} \cos \phi-m g_{\delta} \sin \phi \cos A+m \omega^{2} r \cos \delta(\sin \phi \cos A \sin \delta+ \\
& \cos \phi \cos \delta)+2 m \omega v \sin A \cos \delta .
\end{aligned}
$$

where $f_{T}$ is the applied thrust, $\epsilon$ and $\mu$ are the nozzle heading angles, $D$ is the atmospheric drag, $L$ is lift, $f_{Y}$ is the lateral aerodynamic force, $g_{c}$ is the gravity centripetal component, $g_{\delta}$ is the gravity north component, and $\omega$ is the planet rotational velocity.

\section{Satellite}

The motion of the satellite is said to be purely translational and the related equations are similar to those of the launch vehicle. The equations of motion are written in the reference frame of choice. The external forces are the weight component, with 
gravitational acceleration calculated by the axis-symmetric model of high order and the drag force due to atmospheric resistance. The disturbance forces from the Sun and the Moon are deemed negligible for the proposed analysis. Further discussion about this decision is given bellow. The satellite position is written in spherical coordinates in terms of the body velocities, as shown in Eq. 15 .

$$
\left\{\begin{array}{c}
\dot{r}=-w_{L V L H} \\
\dot{\delta}=\frac{u_{L V L H}}{r} \\
\dot{\lambda}=\frac{v_{L V L H} \sec \delta}{r}
\end{array}\right.
$$

The terms $u_{L V L H}, v_{L V L H}$ and $w_{L V L H}$ are the velocities in the LVLH's $X, Y$ and $Z$ axis, respectively, and are given in a vector form as $\mathrm{V}=\left[u_{L V L H} v_{L V L H} \text { and } w_{L V L H}\right]^{T}$. Thus, the accelerations can be obtained by its time derivative with respect to the inertial frame, defined in Eq. 16:

$$
\dot{V}=\frac{d V}{d t}+\Omega \times V
$$

where vector $\Omega$ is the angular velocity vector of the LVLH in respect to the ECI system. Using the relation $\dot{V}=\frac{F}{m_{\text {sat }}}$ where $F=\left[F_{\mathrm{x}} F_{y} F_{z}\right]^{T}$ is the force vector in the LVLH system, and $m_{\text {sat }}$ the satellite mass, Eq. 17 is obtained:

$$
\left\{\begin{array}{c}
\dot{u}=\frac{F_{x}}{m_{s a t}}+\frac{u_{L V L H} w_{L V L H}-v_{L V L H}^{2} \tan \delta}{r} \\
\dot{v}=\frac{F_{Y}}{m_{s a t}}+\frac{v_{L V L H}\left(w_{L V L H}+u_{L V L H} \tan \delta\right)}{r} \\
\dot{w}=\frac{F_{Z}}{m_{s a t}}-\frac{u_{L V L H}^{2}+v_{L V L H}^{2}}{r}
\end{array}\right.
$$

\section{Disturbance Forces}

In simulations with few orbital periods, usually faster than a day at Earth, the third bodies, Sun and Moon, can be considered as stationary at the ECI frame. Thus, these two bodies are assumed to be fixed in a point at the ECI frame. The Moon generate perturbations at the satellite in the form of forces and torques due to its gravitational field. The Sun perturbs the satellite in the same way, but also generates solar pressure. However, the lower the satellite orbit, the smaller is the contribution of the solar flux on the decay in comparison with the atmospheric drag. For the gravitational disturbance forces, it is possible to show that, when analyzing orbit decay in LEO, the influence of the third body is negligible.

Assuming the idealized model of spherical and homogenous bodies for both the Sun and Moon. The gravitational force for each of the bodies can be found through Eqs. 18 and 19. Where $f_{g S u n}$ is the Sun gravitational force, and $f_{g M o o n}$ is the Moon gravitational force acting on the satellite.

$$
\begin{aligned}
& f_{g_{\text {Sun }}}=-\frac{m_{\text {Sun }} m_{\text {sat }} G_{\vec{r}_{13}}}{r_{13}^{3}} . \\
& f_{g_{\text {Moon }}}=-\frac{m_{\text {Moon }} m_{\text {sat }} G_{\vec{r}_{14}}}{r_{14}^{3}} .
\end{aligned}
$$

where $m_{\text {sat }}$ is the satellite mass, $r_{13}$ is the satellite position vector relative to the Sun center of mass, $m_{\text {Sun }}$ the Sun mass and $G_{\vec{r}_{13}}$ is the Sun gravitational constant. Similarly, $r_{14}$ is the satellite position vector relative to the Moon center of mass, $m_{\text {Moon }}$ is the mass of the Moon and $G_{\vec{r}_{14}}$ is the Moon gravitational constant. 
Hence, $f_{g S \text { Sun }}$ and $f_{g M o o n}$ can be obtained in a reference system of preference, as a function of the reference system used to express the position vectors, and incorporated in the satellite dynamic equations. However, for LEO the distance between the satellite position and the third bodies is big enough to ensure that the magnitude of $f_{g S u n}$ and $f_{g M o o n}$ is small enough that the third body disturbance is negligible.

\section{Gravitational Model}

For the modelling of Earth's gravitational field, a high order axis symmetric model of the planet is utilized. The model employs high order spherical harmonics to account for the measured deviation between the real gravitational field of Earth and the one estimated with a perfectly spherical and homogenous planet model.

The solution for the deviation problem is given, as shown in Tewari (2007), using the gravitational potential function. This potential function is obtained based on the radial distance of the test mass, $r$, and colatitude $\zeta$, as Eq. 20 presents. For the simulations, the test mass can be either the launch vehicle or the satellite.

$$
\Phi(\mathrm{r}, \xi)=\frac{\mathrm{GM}_{\mathrm{T}}}{r}\left(1-\sum_{n=2}^{\infty}\left(\left(\frac{R e}{r}\right)^{n} J_{n} P_{n}(\cos \xi)\right)\right)
$$

where $G, M_{T}$ and $R_{e}$ are the gravitational constant, Earth's mass and radius at the equator, respectively. Further, the $P_{n}$ terms are found from the Legendre polynomial described in Eq. 21 and $J_{n}$ are the Jeffery's constants.

$$
P_{n}(\cos \xi)=\frac{(2 n-1) \cos \xi P_{n-1} \cos \xi-(n-1) P_{n-2} \cos \xi}{n} .
$$

For this work the model is calculated with $n=4$ and the constants are $J_{2}=0.00108263, J_{3}=0.0000254$ and $J_{3}=0.000000161$. Hence, the gravity acceleration can be determined through Eq. 22.

$$
g=-\frac{\partial \Phi^{T}}{\partial \vec{r}}
$$

\section{Aerodynamic Model}

The aerodynamic model of the launch vehicle calculates the atmospheric drag, which depends on the flow regime. The calculated atmospheric drag is function of the drag coefficient, $C_{D}$, the launch vehicle velocity, $v$, the atmospheric air density, $\rho$, and the vehicle cross sectional area, $S$. The utilized drag coefficient is based on the model by Tewari (2007) for a reentry capsule and is a function of the Knudsen number $\left(K_{n}\right)$, Mach number $(M a)$, and Reynolds number $\left(R_{e}\right)$. Thus, the drag on the launch vehicle, $D_{\text {launcher }}$ is defined in Eq. 23.

$$
D_{\text {launcher }}=\frac{1}{2} \rho v^{2} S C_{D}\left(K_{n}, M_{a}, R e\right)
$$

The aerodynamic forces on the satellite are, together with the gravitational force, the most important influence on the satellite decay. The atmospheric drag is the main component of these forces. Thus, the torques due to interaction with the atmosphere were not included in the simulation. For the drag calculation, a nonrotating $1 \mathrm{U}$ CubeSat with the center of mass coincident with the geometric center was considered. The total drag is the sum of the drag on each of the satellite faces point towards the velocity vector. First, the vector dynamic pressure in the ECI frame is calculated through Eq. 24.

$$
\bar{q}_{s a t}=\frac{1}{2} \rho v_{r e l}^{2}
$$


where $\rho$ is the atmospheric density and $v_{\text {rel }}$ is the velocity in the ECI relative to the ECEF. Next, the dynamic pressure is transformed to body axes for each altitude and attitude of the satellite. Finally, the total drag is calculated as the sum of the drag on each face pointing towards the velocity vector. The drag in the face of the satellite, calculated through Eq. 25 , is the product of the dynamic pressure magnitude, $\bar{q}_{\text {sat }}$, the face reference area, $S_{\text {ref }}$ and the drag coefficient, $C_{D}$, which is given by Oltrogge and Leveque (2011) for a $1 \mathrm{U}$ CubeSat and is equal to 2.2 .

$$
D_{\text {face }}=\bar{q}_{s a t} S_{\text {ref }} C_{D}
$$

\section{Propulsive Model}

The propulsive model is only applicable to the launching vehicle, as the satellite is assumed to not have a propulsive system. The vehicle propulsive model considers a continuous and homogeneous burning rate. The thrust is calculated for each stage and is defined as a rectangular pulse, in other words, it goes instantaneously to maximum when the engine is activated and stays in maximum thrust until the end of the burn, when the thrust goes immediately to zero. The thrust is a function of the specific impulse and burn time, as presented in Eq. 26.

$$
T=g_{0} I_{s p} \dot{m}
$$

where $g_{0}$ is the magnitude of the gravity acceleration at sea level and $\dot{m}$ is constant for each stage.

\section{RESULTS}

\section{Launch}

After modeling the launch vehicle and its parameters, the launch of VSB-30M was simulated using the gravity turn maneuver to insert payload into orbit. Table 4 displays ignition, burnout and separation chosen time parameters to achieve the gravity turn maneuver. It is worth noting that those times were chosen with a small optimization procedure, the general idea is that, for a circular orbit, the flight path angle $\phi$ need to be close to zero, hence the rocket was launched with just the first two stages with the times shown in Table 4 and the time where $\phi$ cross the zero was observed. In that way, one knows that the actual $t_{i, 3}$ must be close to this observed time and only small adjustments are necessary to obtain the desired orbit. For the solid rocket, the initial time for the nth stage is $t_{i, n}$, the burning time is $t_{q, n}$, finally the $t_{s, n}$ is the separation time all of them have $n=1,2,3$.

Table 4. Ignition, burnout and separation time for the VSB-30M.

\begin{tabular}{ccccccccc}
\hline$t_{\mathrm{i}, 1}$ [s] & $t_{\mathrm{i}, 2}$ [s] & $t_{\mathrm{i}, 3}$ [s] & $t_{\mathrm{q}, 1}$ [s] & $t_{\mathrm{q}, 2}$ [s] & $t_{\mathrm{q}, 3}$ [s] & $t_{\mathrm{s}, 1}$ [s] & $t_{\mathrm{s}, 2}[\mathbf{s}]$ & $t_{\mathrm{s}, 1}$ [s] \\
\hline 0.0 & 15.0 & 232.7 & 13.5 & 44.0 & 262.7 & 13.5 & 59.0 & 267.7 \\
\hline
\end{tabular}

These times, for the VSB-30M with a solid third stage engine can also be seen in the Fig. 4. In addition, the thrust pattern is evident in the figure.

The initial state for the simulation has the initial velocity $v_{0}=1 \mathrm{~m} / \mathrm{s}$, the initial azimuth $A_{0}=90^{\circ}$, the initial flight path angle $\phi=78^{\circ}$, the initial radius $r_{0}=6378137.0$, and latitude and longitude are those of the CLA.

The altitude through time is shown in Fig. 5 , it is clear that the launch succeeds in placing the payload in an eccentric orbit with perigee of $6.591807 \cdot 10^{3} \mathrm{~km}$ and an apogee of $7.055137 \cdot 10^{3} \mathrm{~km}$. However, this is not the desired circular orbit, which could be achieved by means of a more precise selection of the launching times and initial conditions, but, since this is an optimization problem on its own, it won't be treated here. 


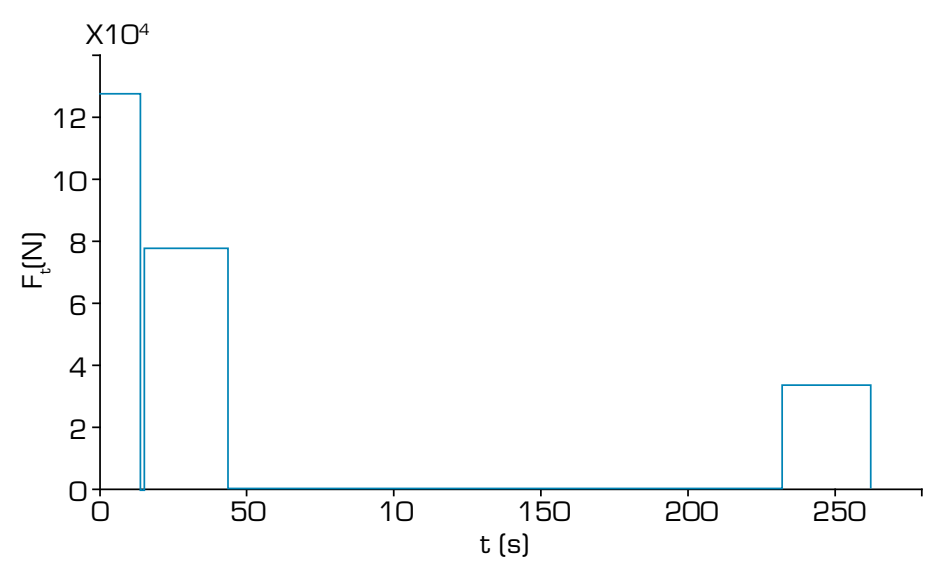

Figure 4. Thrust through time.

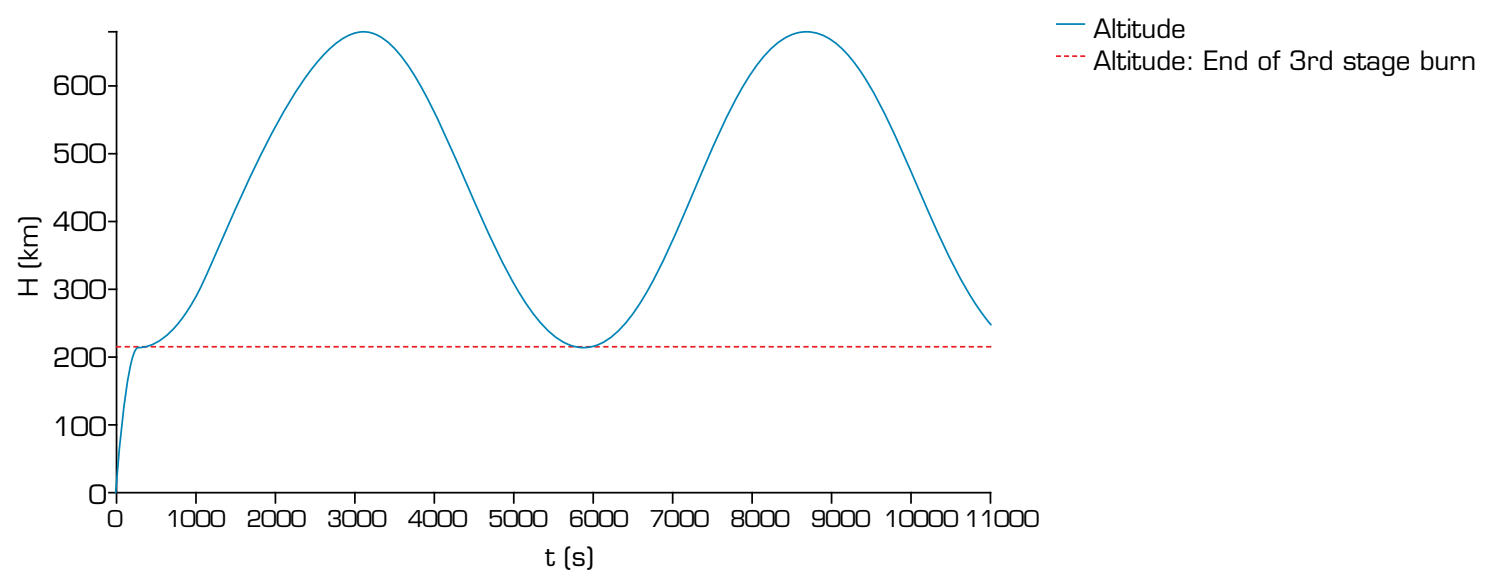

Figure 5. Altitude through time using the solid engine.

The $\phi$ angle is shown in the Fig. 6, evidencing the orbit insertion approach. It can be noticed the final flight path is close to zero, as desired.

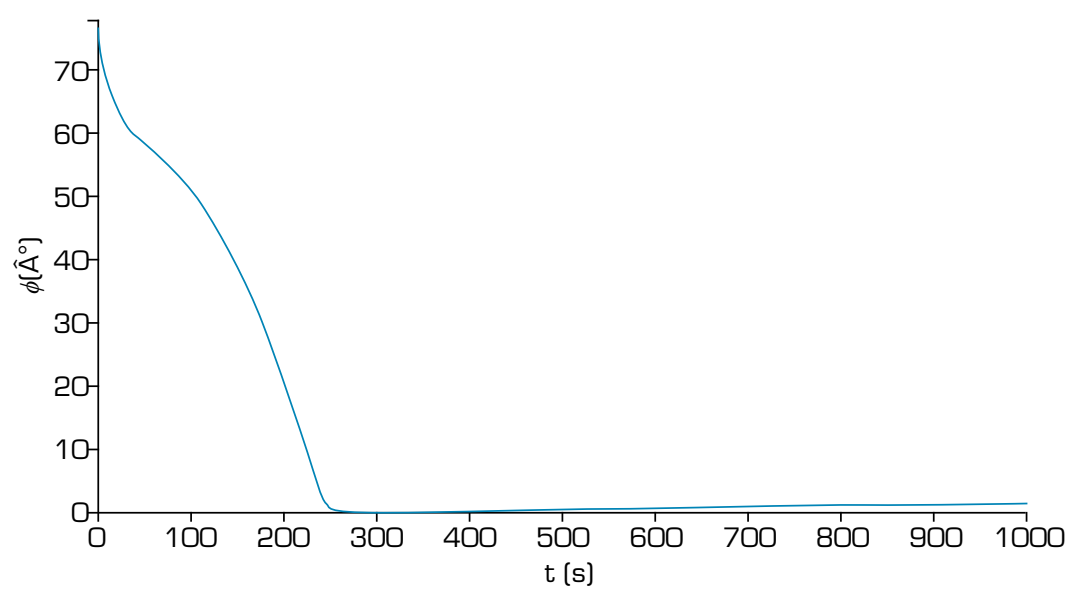

Figure 6. Flight path angle through time using the solid engine. 
A second launch simulation, for the third stage with a liquid engine was performed. It is worth noting that the objective of the simulations is to show that an orbit insertion can be achieved with both third stages and should not be used as a matter of comparison between the two proposed modifications. For this rocket, the orbit insertion is achieved and its altitude through time is shown in Fig. 7 .

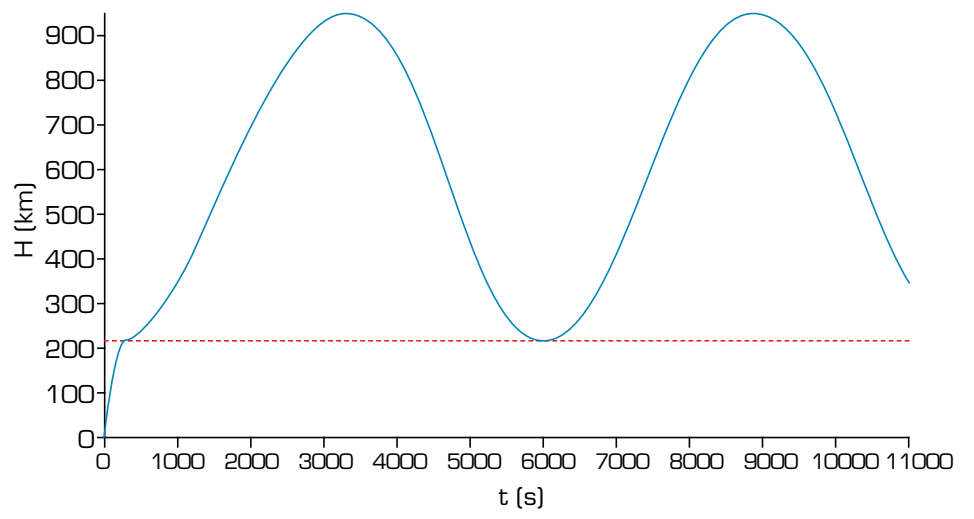

- Altitude

--- Altitude: End of 3rd stage burn

Figure 7. Altitude through time using the liquid engine.

The same initial conditions as the previous launch were used. The times intervals are the same ones described in Table 4 . The achieved orbit, seen in Fig. 8 and 9, has a higher eccentricity with a perigee of $6.59 \cdot 10^{3} \mathrm{~km}$ and an apogee of $7.32 \cdot 10^{3} \mathrm{~km}$. The flight path angle follows the same approach as described earlier.

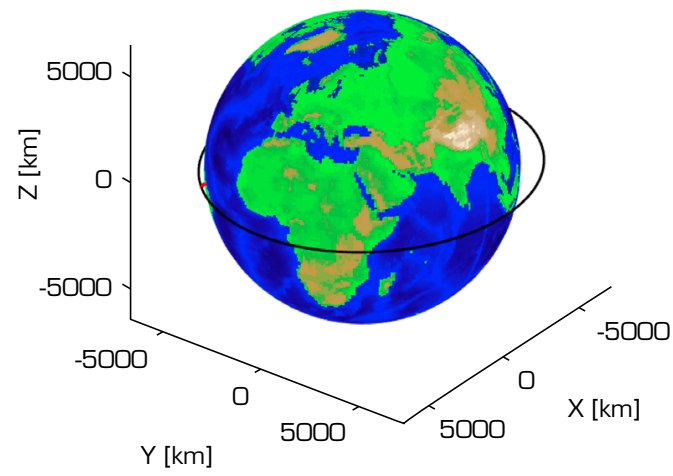

Figure 8. Three-dimensional orbit visualization.

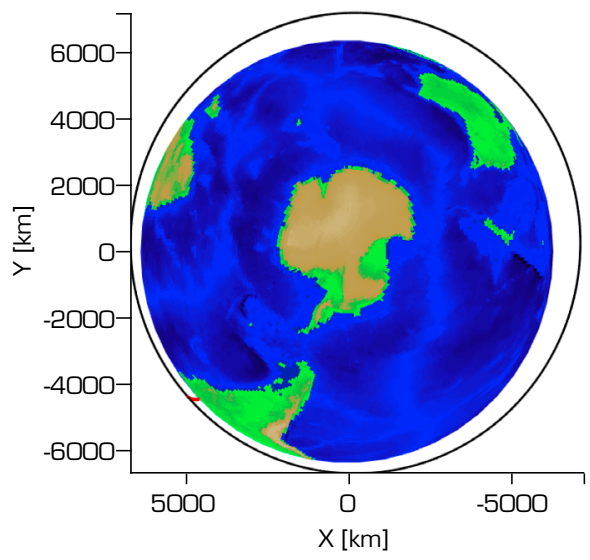

Figure 9. Orbit and launcher trajectory plan view. 


\section{Orbit propagation}

The results obtained from the launch vehicle simulation were used as input for the satellite orbit simulation to evaluate the mission lifetime of a CubeSat at this altitude and, consequently, the feasibility of such a mission. The method for this evaluation was to simulate the satellite orbit until it entered a condition of decay where it did not gained altitude by oscillating, considering that as its reentry and consequent end of lifetime. A second simulation was performed in order to evaluate the impact of small altitude changes (an increase of 7.9\%) in the orbit lifetime. Table 5 presents the initial conditions for both simulations.

Table 5. Initial condition in Keplerian elements.

\begin{tabular}{cccccc}
\hline Simulation & Altitude & Inclination & Eccentricity & Mean anomaly & $\begin{array}{c}\text { Right ascension of } \\
\text { the ascending node }\end{array}$ \\
\hline First & $213 \mathrm{~km}$ & $2.33^{\circ}$ & 0 & $0^{\circ}$ & $0^{\circ}$ \\
Second & $230 \mathrm{~km}$ & $2.33^{\circ}$ & 0 & $0^{\circ}$ & $0^{\circ}$ \\
\hline
\end{tabular}

The results show a mission lifetime of $60 \mathrm{~h}$, as displayed in Fig. 10. The oscillations in altitude indicate the increase in the orbit eccentricity due to the atmospheric drag. Thus, the orbit becomes elliptical. At the end of its lifetime (around $60 \mathrm{~h}$ ), the spacecraft begins a constant reentry phase.

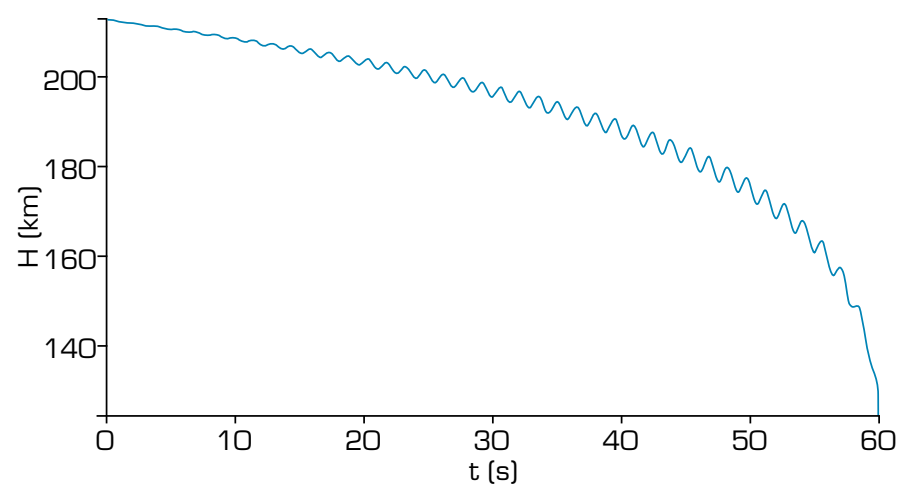

Figure 10. Altitude through time for the first case.

The specific orbital energy variation as a function of time corroborates the previous results, as Fig. 11 indicates. The energy variation is not linear, since the decrease in altitude increases drag, thus increasing the rate of decay. The negative specific energy also indicates an elliptical orbit (positive indicates hyperbolic).

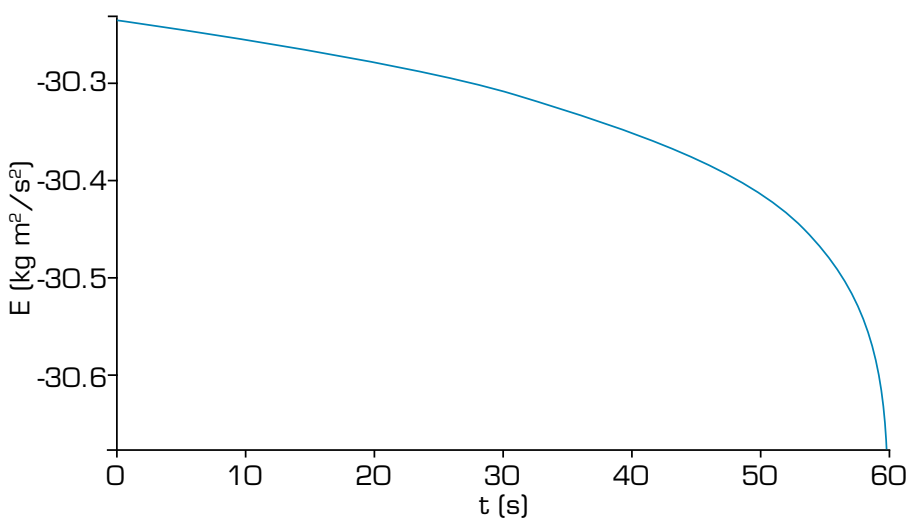

Figure 11. Specific orbital energy through time for the first case. 
Figure 12 shows the altitude through time for the second case, which indicates an increase of $93.3 \%$ in mission lifetime from 60 to $116 \mathrm{~h}$

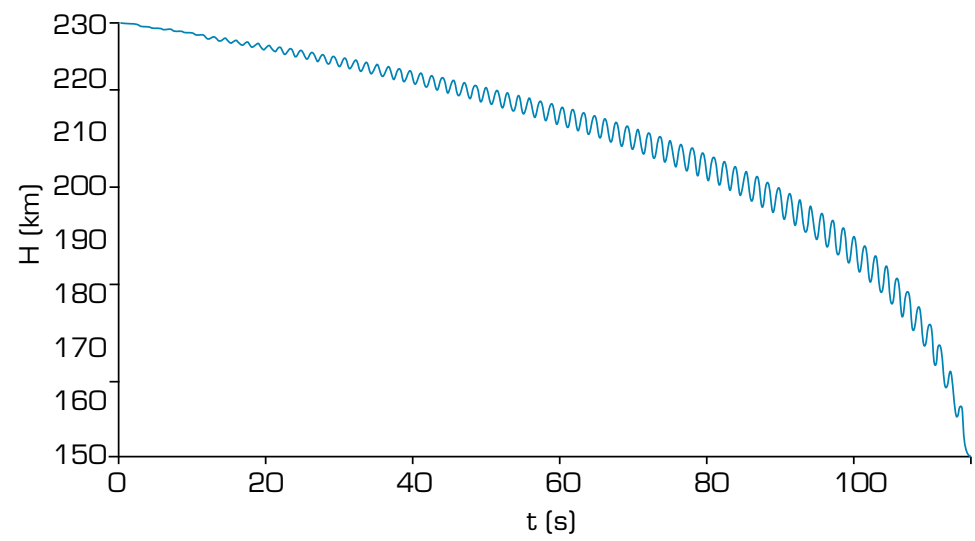

Figure 12. Altitude through time for the second case.

\section{CONCLUSION}

Both modified VSB-30s proposals were successful in reaching the necessary altitude and velocity to insert a satellite into orbit, changing its original profile from suborbital launcher to orbital launcher. The satellite decay simulations show that short time space missions are feasible at the attained orbit, supporting the proposed modifications.

In addition, a small change of $17 \mathrm{~km}$ in the orbit altitude resulted in a mission lifetime increase of $93.3 \%$, indicating that further studies could improve the mission lifetime significantly adopting optimal trajectory, closed-loop control systems or propulsive systems in the satellite. In addition, a more detailed analysis of the third stage modifications could result in both better structural ratios and performance.

The fact that Brazil has mature solid rockets technology corroborates with the feasibility of the proposed modifications and mission. Moreover, when available, liquid engines would allow taking advantage of its characteristics such as precise orbit insertion, burn control, and higher specific impulses, increasing the range of possible missions.

Furthermore, modifications of a national vehicle could collaborate with the development of the Brazilian space industry, increasing the country's autonomy in this segment.

\section{AUTHORS' CONTRIBUTION}

Conceptualization: Silva AL; Slongo AG; Moraes DD; Mantovani LQ; Venturini MS; Data Curation: Mantovani, LQ; Venturini MS; Moraes DD; Formal Analysis: Slongo AG; Mantovani LQ; Sehnem RM; Silva AL; Investigation: Slongo AG; Mantovani LQ; Moraes DD; Methodology: Slongo AG; Mantovani LQ; Sehnem RM; Project Administration: Silva AL; Mantovani LQ; Slongo AG; Moraes DD; Resources: Slongo AG; Software: Silva AL; Mantovani LQ; Slongo AG; Sehnem RM; Martos JFA; Supervision, Silva AL; Martos JFA; Validation: Silva AL; Martos JFA; Visualization: Mantovani LQ; Writing - Original Draft: Mantovani LQ; Slongo AG; Moraes DD; Venturini MS.

\section{DATA AVAILABILITY STATEMENT}

All the datasets were generated and analyzed during the current study. 


\section{FUNDING}

This research received no specific grant from any funding agency in the public, commercial, or not-for-profit sectors.

\section{ACKNOWLEDGEMENTS}

The authors thank the Organization Committee of II Congresso Aeroespacial Brasileiro, to the students of the first and second classes of the Aerospace Engineering course at the Federal University of Santa Maria for the discussions and support.

\section{REFERENCES}

[AEB] Agência Espacial Brasileira (2020) NanosatC-BR2 passa por testes e está pronto para ser lançado no fim do ano. [accessed Aug. 21 2020]. https://www.gov.br/aeb/pt-br/assuntos/noticias/nanosatc-br2-passa-por-testes-e-esta-pronto-para-ser-lancadono-fim-do-ano

[CGEE] Centro de Gestão e Estudos Estratégicos (2018) CubeSats. Brasília: CGEE. [accessed Aug. 21 2020]. https://www.cgee. org.br/documents/10195/734063/CGEE_resumoexecutivo_CubeSats_Web.pdf

[DCTA] Departamento de Ciência e Tecnologia Aeroespacial (2019) Centro de Lançamento de Alcântara. Força Aérea Brasileira. [accessed Aug. 21 2020]. http://www2.fab.mil.br/cla/index.php/historias

[ITA] Instituto Tecnológico da Aeronáutica (2020) Kick-off do projeto ITASAT 2. [accessed Aug. 21 2020]. http://www.ita.br/ noticias/kickoffdoprojetoitasat2

Costa FS, Vieira R (2010) Preliminary analysis of hybrid rockets for launching nanosats into LEO. J Braz Soc Mech Sci Eng 32(4):502-509. https://doi.org/10.1590/S1678-58782010000400012

Garcia A, Yamanaka SSC, Barbosa AN, Bizarria FCP, Jung W, Scheuerpflug F (2011) VSB-30 sounding rocket: history of flight performance. J Aerosp Technol Manag 3(3):325-330. https://doi.org/10.5028/jatm.2011.03032211

Inatani Y, Ohtsuka H (2018) SS-520 Nano satellite launcher and its flight result. Paper presented 32nd Annual AIAA/USU Conference on Small Satellites. AIAA; Logan, Utah, United States.

Isakowitz SJ, Hopkins Junior JP, Hopkins JB (1999) International reference guide to space launch systems. Reston: AIAA.

Ley W, Wittmann K, Hallmann W (2009) Handbook of space technology. New York: John Wiley \& Sons. https://doi. org/10.2514/4.867019

Niederstrasser C (2018) Small launch vehicles-a 2018 state of the industry survey. Paper presented 32nd Annual AIAA/USU Conference on Small Satellites. AIAA; Logan, Utah, United States.

Niederstrasser C, Madry S (2020) New launchers for small satellite systems. In: Pelton JN, Madry S, editors. Handbook of Small Satellites: Technology, Design, Manufacture, Applications, Economics and Regulation. Cham: Springer. p. 1-14. https://doi. org/10.1007/978-3-030-20707-6_21-1

O’Donnell K, Richardson G (2020) Small satellite trending \& reliability 2009-2018. Paper presented 34th Annual Small Satellites Conference. AIAA; Logan, Utah, United States. 
Oltrogge D, Leveque K (2011) An evaluation of CubeSat orbital decay. Paper presented 25th Annual AIAA/USU Conference on Small Satellites. AIAA; Logan, Utah, United States.

Palmerio AF (2017) Introdução à tecnologia de foguetes. São José dos Campos: SindCT.

Sato LHS, Costa LEVL, Fulindi JB, Santos HA, Paula LS, Oliveira EHS, Azevedo JG, Crucioli BA, Vieira DG, Carrara V, et al. (2019) The ITASAT-the lessons learned from the mission concept to the operation. Paper presented 33rd Annual AIAA/USU Conference on Small Satellites. AIAA; Logan, Utah, United States.

Silva RGA, Damilano JG, Azevedo JLF (2013) A sensitivity investigation on the aeroelastic dynamic stability of slender spinning sounding rockets. J Aerosp Technol Manag 5(1):15-26. https://doi.org/10.5028/jatm.v5i1.192

Sutton GP, Biblarz O (2001) Rocket propulsion elements. New York: John Wiley \& Sons.

Tewari A (2007) Atmospheric and Space Flight Dynamics: Modeling and Simulation with MATLAB and Simulink. Basel: Birkhäuser.

Wekerle T, Pessoa Filho JB, Costa LEVL, Trabasso LG (2017) Status and trends of smallsats and their launch vehicles - an upto-date review. J Aerosp Technol Manag 9(3):269-286. https://doi.org/10.5028/jatm.v9i3.853

Wie B (2008) Space vehicle dynamics and control. Reston: AIAA. https://doi.org/10.2514/4.860119 